\title{
Serum 205 proteasome is elevated in patients with renal cell carcinoma and associated with poor prognosis
}

\author{
M de Martino ${ }^{1,5}$, K Hoetzenecker $^{2,5}$, HJ Ankersmit ${ }^{2}$, GA Roth ${ }^{3}$, A Haitel ${ }^{4}$, M Waldert' and T Klatte ${ }^{*, 1}$ \\ 'Department of Urology, Medical University of Vienna, Währinger Gürtel 18-20, Vienna 1090, Austria; 'Department of Thoracic Surgery, \\ Medical University of Vienna, Währinger Gürtel 18-20, Vienna 1090, Austria; ${ }^{3}$ Department of Anesthesiology, General Intensive Care and Pain Medicine, \\ Medical University of Vienna, Währinger Gürtel 18-20, Vienna 1090, Austria; ${ }^{4}$ Department of Clinical Pathology, Medical University of Vienna, \\ Währinger Gürtel 18-20, Vienna 1090, Austria
}

BACKGROUND: To date, no reliable serum marker for clear cell renal cell carcinoma (CCRCC) is available. The aim of this study was to evaluate the putative significance of circulating $20 \mathrm{~S}$ proteasome levels.

METHODS: Preoperative 20S proteasome serum levels were determined in II3 CCRCC patients and I5 healthy controls by a sandwich enzyme-linked immunosorbent assay. Associations with CCRCC, pathological variables, disease-specific survival (DSS), and response to sunitinib were evaluated.

RESULTS: Median $20 S$ proteasome levels were higher in CCRCC patients than in healthy controls $\left(4.66 \mathrm{vs} \mathrm{I.52} \mu \mathrm{g} \mathrm{ml}^{-1}, \mathrm{P}<0.000 \mathrm{I}\right)$. The area under the receiver operating characteristics curve curve was $87.1 \%$. The $20 \mathrm{~S}$ proteasome levels were associated with symptoms $(P=0.0008)$, distant metastases $(P=0.00 \mathrm{II})$, grade $(P=0.0247)$, and necrosis $(P=0.0462)$. The $20 \mathrm{~S}$ proteasome levels were identified as a prognostic factor for DSS in both univariable (hazards ratio I.2I, $P<0.001$ ) and multivariable (hazards ratio I. I7, $P=0.0015)$ survival analysis. In patients responding to sunitinib, 20 S proteasome levels were lower than in patients with stable disease and progressive disease.

CONCLUSION: This study demonstrates for the first time that increased 20S proteasome levels are associated with CCRCC, advanced disease, and poor prognosis. Routine use of this marker may allow better diagnosis, risk stratification, risk-adjusted follow-up, and identification of patients with a greater likelihood of response to targeted therapy.

British Journal of Cancer (2012) I 06, 904-908. doi:I0.1038/bjc.2012.20 www.bjcancer.com

Published online 31 January 2012

(c) 2012 Cancer Research UK

Keywords: 20 S proteasome; renal cancer; hypoxia; diagnosis; prognosis

More than 250000 patients are newly diagnosed with renal cell carcinoma (RCC) worldwide annually (Ferlay et al, 2010). Although the mortality rate has stabilised or declined in most countries since the 1990s, more than 100000 deaths are attributed to the disease every year (Ferlay et al, 2010).

During the last decades, our understanding of RCC biology has improved considerably. Several subtypes have been delineated, including clear cell RCC (CCRCC), papillary, chromophobe, collecting duct, and unclassified RCC. Additionally, subtypespecific molecular pathways and the genetic basis of these pathways have been characterised (Klatte and Pantuck, 2008). This knowledge led to the development of novel systemic therapies that have changed the therapeutic landscape (Gore and Larkin, 2011; Powles et al, 2011; Procopio et al, 2011). Research showed that the biology of CCRCC is in part driven by the hypoxiainducible factor (HIF) protein family, the von Hippel-Lindau $(V H L)$ gene, and the ubiquitin-proteasome pathway (Klatte and Pantuck, 2008). HIF serves as a transcription factor of hypoxiainducible genes such as vascular endothelial growth factor (VEGF),

*Correspondence: Dr T Klatte; E-mail: tobias.klatte@gmx.de

${ }^{5}$ These authors contributed equally to this work.

Received I 5 November 20 I ; accepted 9 January 20 I2; published online 31 January 2012 platelet-derived growth factor, transforming growth factor- $\alpha$, carbonic anhydrase IX, and the chemokine receptor CXCR4 (Klatte and Pantuck, 2008). HIF and its downstream targets have been identified as key regulators of CCRCC progression (Klatte et al, 2007b; Klatte and Pantuck, 2008).

Under conditions of normoxia and wild-type VHL, HIF is labelled with ubiquitin and subsequently degraded via the proteasome (Corn, 2007). In CCRCC, the ubiquitin-proteasome pathway is frequently impacted due to hypoxia and VHL loss. A constitutively increased proteasome activity has been observed in CCRCC, which correlates with proliferation (Kanayama et al, 1991). Despite HIF, peptides and proteins involved in basic cellular processes, such as cell cycle regulation, apoptosis, signal transduction, transcriptional activation, antigen processing, and cancer cachexia are processed via the proteasome (Tisdale, 2003; Roth et al, 2005). This multi-catalytic protease complex is located in both the cytoplasm and the nucleus. Its $20 \mathrm{~S}$ subunit constitutes the core of the proteasome. Each core of a 20S subunit is further assembled by the $19 \mathrm{~S}$ regulatory subunit, which together form the $26 S$ proteasome. Upon translocation of ubiquitinated proteins into the proteasome, the target protein undergoes ATP-dependent degradation (Voges et al, 1999). Importantly, function of the $20 \mathrm{~S}$ proteasome can be reversibly inhibited by bortezomib (Groll et al, 2006). In metastatic RCC, clinical responses have been observed following administration of this agent (Kondagunta et al, 2004). 
To date, no reliable diagnostic or prognostic serum marker for CCRCC is available. As $20 \mathrm{~S}$ proteasome levels can be detected in the serum, it may represent a novel biomarker for this entity, which could facilitate diagnosis and postoperative risk-group assessment, and may help in identifying patients that most likely respond to targeted agents. To date, however, no data have been reported. In the present pilot study, we have analysed preoperative 20 S proteasome serum levels in CCRCC patients and healthy controls, using a validated enzyme-linked immunosorbent assay (Roth et al, 2005; Szerafin et al, 2008). Further, we have investigated the association of $20 \mathrm{~S}$ proteasome serum levels with clinical and pathological factors, response to targeted agents, and survival, to determine its potential predictive and prognostic significance.

\section{PATIENTS AND METHODS}

\section{Study population}

This study was reviewed and approved by the institutional review board of the Medical University of Vienna, Austria. A total of 128 peripheral venous blood samples (113 with CCRCC, 15 healthy blood donors) were obtained at the Department of Urology, Medical University of Vienna, Austria, between February 2008 and July 2009. To minimise protein degradation, the samples were processed immediately. Following upright storage for $10 \mathrm{~min}$, serum was obtained after centrifugation $(1800 \mathrm{~g}, 10 \mathrm{~min})$ and stored at $-80{ }^{\circ} \mathrm{C}$ until analysis. The 113 CCRCC serum samples were selected out of 153 preoperative serum samples, which were collected from consecutive patients with an RCC-suspicious renal tumour scheduled for partial or radical nephrectomy. The excluded 40 serum samples refer to patients with non-CCRCC $(n=22)$, bilateral RCC $(n=2)$, hereditary RCC $(n=1)$, or benign renal tumours $(n=15)$.

Baseline clinical and pathological data were abstracted from a prospectively maintained institutional review board-approved kidney cancer database, and included age, gender, symptoms, 2009 TNM stage (Sobin et al, 2009), Fuhrman grade, pathological tumour size, and tumour necrosis. Diagnosis of CCRCC, the Fuhrman grade, tumour necrosis, and TNM stage was confirmed by one dedicated uro-pathologist (AH).

Radical and partial nephrectomy was performed in 59 (52\%) and $54(48 \%)$ patients, respectively. None of the patients received adjuvant therapy. All patients with non-metastatic disease were followed systematically at our outpatient clinic according to the established guidelines (Ljungberg et al, 2007). Of the 32 patients who developed or presented with metastatic disease, the first-line therapy comprised sunitinib ( $50 \mathrm{mg}$ po $\mathrm{q} 24 \mathrm{~h}, n=22$ ), temsirolimus $(25 \mathrm{mg}$ iv q7d, $n=2)$, metastasectomy $(n=3)$, or best supportive care $(n=5)$. In patients receiving sunitinib and temsirolimus, imaging was repeated after two cycles and in 8-week-intervals, respectively. Response was evaluated according to the RECIST criteria. Median follow-up for the patients alive was 30 months (interquartile range, 14 months). Out of 25 patients, who were deceased at last follow-up, 21 died from CCRCC.

\section{Serum 20S proteasome measurements}

The $20 \mathrm{~S}$ proteasome serum levels were determined by a sandwich enzyme-linked immunosorbent assay (Roth et al, 2005; Szerafin et al, 2008). Microtitration plates were coated overnight with the mouse monoclonal antibody to the $20 \mathrm{~S}$ proteasome subunit a6 (1:4500 in carbonate buffer, $\mathrm{pH}$ 9.6, Affiniti Research Products Ltd, Exeter, UK). Remaining binding sites were blocked with $0.5 \%$ fetal calf serum in PBS, $\mathrm{pH}$ 7.4. Serum samples were diluted $1: 20$ and applied to each well for $3 \mathrm{~h}$ at room temperature. Standard curves were established using 20S proteasome (Affiniti Research
Products Ltd) in a concentration from 5000 to $78 \mathrm{ng} \mathrm{ml}^{-1}$ (Affiniti Research Products Ltd). After a washing step, a rabbit polyclonal antibody to $20 \mathrm{~S}$ proteasome $\alpha / \beta$-subunits was added for $2 \mathrm{~h}$ at room temperature (Affiniti Research Products Ltd). Following another washing step, a peroxidase-conjugated mouse anti-rabbit IgG (Jackson ImmunoResearch, West Grove, PA, USA) was used for the detection of the antigen. The bound antibodies were detected with tetramethylbenzidine as substrate. The reaction was stopped with sulphuric acid and OD values were determined at $450 \mathrm{~nm}$. To exclude the possibility of non-specific binding, we tested bovine serum albumin as control protein instead of $20 \mathrm{~S}$ proteasome, and did not observe any positive reaction.

\section{Statistical analysis}

The continuous data were tested for normal distribution using the Kolmogorov-Smirnov test and were found to be not normally distributed. Thus, associations of $20 \mathrm{~S}$ proteasome serum levels with clinical and pathological parameters were assessed with non-parametric Kruskal - Wallis rank sum tests. Correlations were determined using the non-parametric Spearman's rank correlation. The diagnostic performance of $20 \mathrm{~S}$ proteasome serum levels was analysed with a receiver operating characteristics curve (ROC). The ROC curve is a plot of sensitivity vs 1-specificity for all possible cut-point values. The area under the ROC curve was applied to evaluate the diagnostic accuracy. The 95\% confidence intervals $(95 \%$ CI) were calculated according to the DeLong method.

To assess 20S proteasome serum levels as a prognostic marker, associations with disease-specific survival (DSS) time were assessed. DSS was calculated from the date of surgery. A cut point for prognostic sub-stratification was identified with recursive partitioning-based survival tree analysis. Univariable and multivariable Cox proportional hazards models were fit to evaluate the relative impact of variables on DSS. Because of the low number of events, an over-fit bias was likely to occur in multivariable analysis. Thus, not all relevant prognostic factors were included as a single variable. Rather, the main prognostic factors ( T, N, M stage, size, grade, and necrosis) were combined as SSIGN score (Frank et al, 2002). Predictive accuracies were assessed by concordance index. The likelihood ratio test was applied to compare predictive accuracies between nested models. Statistical analyses were all performed with the freely available statistical package R-2.10.1 (http://cran.r-project.org/) using the Epi, Design and survival libraries. A $P$-value $<0.05$ was considered statistically significant.

\section{RESULTS}

\section{Association with CCRCC}

Overall median serum level of $20 \mathrm{~S}$ proteasome was $4.21 \mu \mathrm{g} \mathrm{ml}^{-1}$ (interquartile range, 4.29), and the levels differed significantly between the CCRCC patients (median $4.66 \mu \mathrm{g} \mathrm{ml}^{-1}$ ) and healthy controls $\left(1.52 \mu \mathrm{g} \mathrm{ml}^{-1}, P<0.0001\right)$. The area under the ROC curve under the ROC curve was $87.1 \%$ (95\% CI, 77.8-96.5). At the optimal cut point, the sensitivity and the specificity reached $87.6 \%$ (95\% CI, 80.1-93.1) and 73.3\% (95\% CI, 44.9-92.0), respectively (Figure 1).

\section{Association with clinical and pathological variables}

Characteristics of CCRCC patients and associations with 20S proteasome serum levels are shown in Table 1. The 20S proteasome serum levels were higher in patients with CCRCCassociated symptoms $(P=0.0008)$, with distant metastases $(P=0.0011)$, higher Fuhrman grades $(P=0.0247)$, and necrotic tumours $(P=0.0462)$. Additionally, there was a statistically 


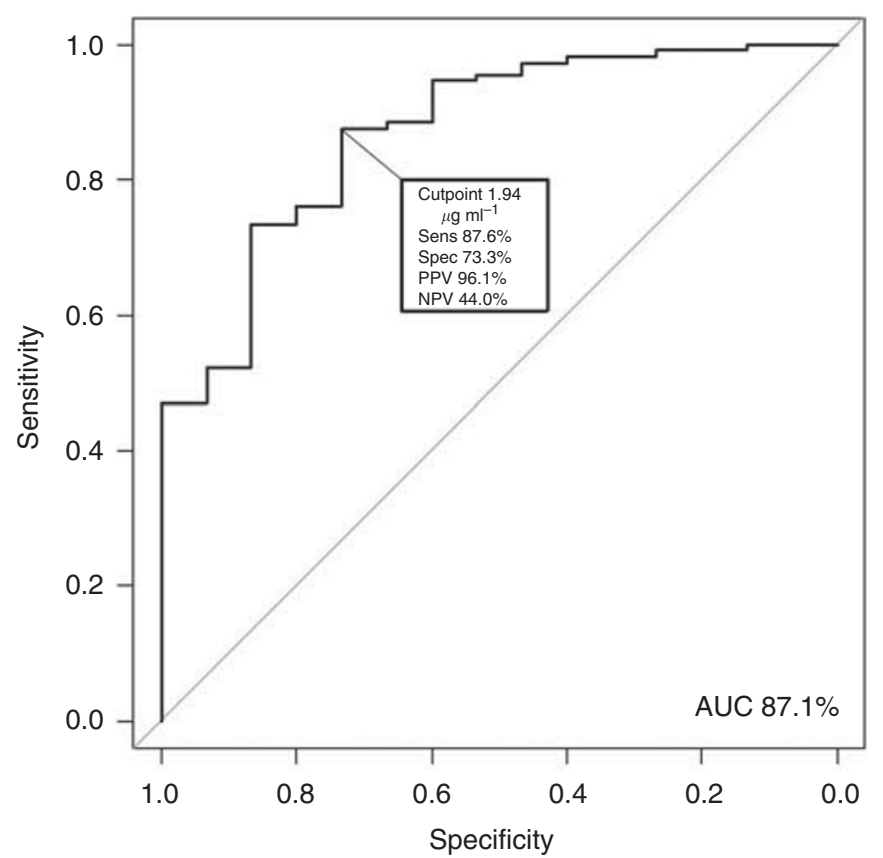

Figure I Accuracy of 205 proteasome serum levels for diagnosis of CCRCC. The area under the ROC curve (AUC) was 87.1\%. A cut point of $1.94 \mu \mathrm{g} \mathrm{ml}^{-1}$ showed the highest diagnostic accuracy.

Table I Descriptive statistics of CCRCC patients and associations with $20 \mathrm{~S}$ proteasome serum levels

\begin{tabular}{|c|c|c|c|c|c|}
\hline \multirow[b]{2}{*}{ Variable } & \multicolumn{2}{|c|}{ RCC population } & \multicolumn{2}{|c|}{$20 S$ proteasome levels } & \multirow[b]{2}{*}{$P$-value } \\
\hline & No. & $\%$ & Median & IQR & \\
\hline Gender & & & & & 0.1832 \\
\hline Female & 38 & 34 & 5.40 & 4.54 & - \\
\hline Male & 75 & 66 & 4.30 & 4.27 & - \\
\hline Symptoms & & & & & 0.0008 \\
\hline No & 85 & 75 & 4.03 & 3.69 & - \\
\hline Yes & 28 & 25 & 7.40 & 7.51 & - \\
\hline$T$ stage & & & & & 0.0729 \\
\hline PTI-2 & 54 & 48 & 4.00 & 4.19 & - \\
\hline PT3 & 59 & 52 & 5.17 & 4.27 & - \\
\hline$N$ stage & & & & & 0.2139 \\
\hline $\mathrm{pN} \times / \mathrm{NO}$ & 109 & 96 & 4.65 & 4.19 & - \\
\hline $\mathrm{pNI}$ & 4 & 4 & 7.04 & 2.84 & - \\
\hline M stage & & & & & 0.0011 \\
\hline MO & 90 & 80 & 4.21 & 3.91 & - \\
\hline MI & 23 & 20 & 7.43 & 7.05 & - \\
\hline Fuhrman grade & & & & & 0.0247 \\
\hline Grade I-2 & 82 & 73 & 4.29 & 4.01 & - \\
\hline Grade 3-4 & 31 & 27 & 6.20 & 6.11 & - \\
\hline Tumour necrosis & & & & & 0.0462 \\
\hline No & 61 & 54 & 3.98 & 4.05 & - \\
\hline Yes & 52 & 46 & 5.27 & 4.20 & - \\
\hline SSIGN & & & & & 0.0050 \\
\hline $0-2$ & 51 & 45 & 3.88 & 4.03 & - \\
\hline $3-7$ & 40 & 35 & 4.65 & 3.47 & - \\
\hline 8 or greater & 22 & 19 & 7.39 & 7.04 & - \\
\hline
\end{tabular}

Abbreviations: $C C R C C=$ clear cell renal cell carcinoma; $I Q R=$ interquartile range: $\mathrm{SSIGN}=\mathrm{T}, \mathrm{N}, \mathrm{M}$ stage, size, grade, and necrosis. significant correlation with tumour size $(\rho=0.27, P=0.004)$. The $20 \mathrm{~S}$ proteasome serum levels were not associated with gender $(P=0.1832)$ and $\mathrm{N}$ stage $(P=0.2139)$, and did not correlate with age $(\rho=0.08, P=0.3981)$. Patients with higher $\mathrm{T}$ stages tended to have higher $20 \mathrm{~S}$ proteasome serum levels, although this difference did not reach statistical significance $(P=0.0729)$.

\section{Association with DSS}

Univariable Cox proportional hazards analysis showed a hazard ratio of 1.21 (95\% CI, $1.11-1.31)$, indicating that the risk of death from CCRCC increased by $21 \%$ by each $1 \mu \mathrm{g} \mathrm{ml}^{-1}$ increase in $20 \mathrm{~S}$ proteasome serum levels $(P<0.0001)$. For graphical illustration with Kaplan-Meier survival estimates, a recursive partitioningbased survival tree analysis was carried out. A cut point of $7.24 \mu \mathrm{g} \mathrm{ml}^{-1}$ was identified for further prognostic sub-stratification. Using this cut point, there were 85 patients $(75 \%)$ with low levels and $28(25 \%)$ with high levels. The 1- and 3-year survival probabilities ( \pm s.e.) for patients with low levels $v s$ high levels were $98 \pm 2 \%$ vs $67 \pm 9 \%$, and $90 \pm 4 \%$ vs $46 \pm 11 \%$, respectively $(P<0.0001$, Figure 2$)$. In the multivariable model, SSIGN score and $20 \mathrm{~S}$ proteasome serum levels were retained as independent prognostic factors (Table 2). The predictive accuracy of SSIGN

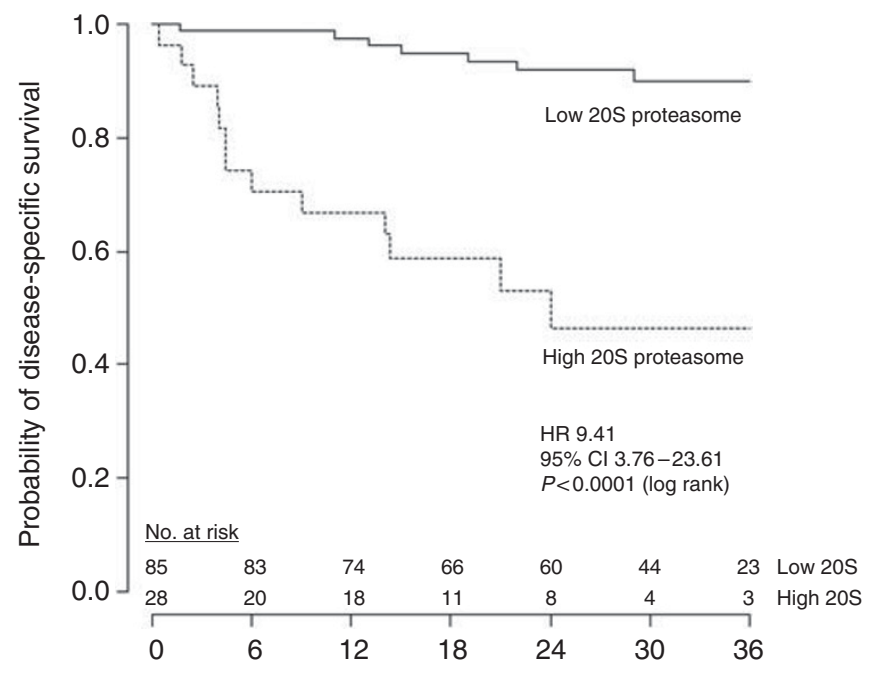

Figure 2 Association of 20S proteasome serum levels with DSS. Patients with high $20 \mathrm{~S}$ proteasome serum levels had a $9.4 \mathrm{I}$-fold increased risk of death from CCRCC, compared with patients with low 205 proteasome serum levels. The optimal cut point of $7.24 \mu \mathrm{g} \mathrm{ml}^{-1}$ was identified through recursive partitioning.

Table 2 Multivariable Cox proportional hazards models

\begin{tabular}{lccrc}
\hline & HR & $\mathbf{9 5 \%} \mathbf{~ C l}$ & P-value & C-index (\%) \\
\hline Model 1 & & & & \\
$\quad$ SSIGN score & 1.69 & $1.39-2.06$ & $<0.0001$ & 92.5 \\
20S proteasome continuous & 1.17 & $1.06-1.28$ & 0.0015 & \\
Model 2 & & & & \\
SSIGN score & & & & \\
20S proteasome categorical & 4.03 & $1.33-1.92$ & $<0.0001$ & 93.2 \\
\end{tabular}

Abbreviations: $\mathrm{Cl}=$ confidence intervals; $\mathrm{C}$-index= concordance index; $\mathrm{HR}=\mathrm{ha}$ zards ratio; $\mathrm{SSIGN}=\mathrm{T}, \mathrm{N}, \mathrm{M}$ stage, size, grade, and necrosis. The models included SSIGN score and 20S proteasome serum level as continuous (model I) or categorical variable (model 2). In both models, $20 \mathrm{~S}$ proteasome serum levels were retained as independent prognostic factor. The C-index increased significantly from 91.4 to $92.5 \%$ and $93.2 \%$, respectively, after the 20 S proteasome serum level was introduced in the model. 
Table $320 \mathrm{~S}$ proteasome serum levels decreased with increasing response category

\begin{tabular}{lrrrr}
\hline & & & \multicolumn{2}{c}{ 20S proteasome levels } \\
\cline { 4 - 5 } Response category & No. & $\%$ & Median & IQR \\
\hline Progressive disease & 6 & 27 & 8.18 & 7.40 \\
Stable disease & 11 & 50 & 6.20 & 3.80 \\
Partial response & 5 & 23 & 3.65 & 0.84 \\
\hline
\end{tabular}

Abbreviation: $\mathrm{IQR}=$ interquartile range.

score alone was $91.4 \%$. It increased significantly to $92.5 \%$ $(P=0.0025)$ with continuous, and to $93.2 \%(P=0.0030)$ with dichotomised $20 \mathrm{~S}$ proteasome serum levels.

\section{Association with response to systemic therapy}

In 24 patients with metastatic disease, a systemic first-line therapy was administered. Both patients, who had received temsirolimus, had progressive disease at first re-imaging and died from CCRCC after 4 and 5 months, respectively. Of the 22 patients, who had received sunitinib, there were $6(27 \%)$ with progressive disease, $11(50 \%)$ with stable disease, and $5(23 \%)$ who exhibited a partial response. The $20 \mathrm{~S}$ proteasome serum levels decreased with increasing response category (Table 3). However, due to the low numbers of patients, this difference did not reach statistical significance $(P=0.2058)$.

\section{DISCUSSION}

This is the first study, which evaluated the putative significance of preoperative $20 \mathrm{~S}$ proteasome serum levels in CCRCC. We found that this marker is elevated in patients with CCRCC, as compared with healthy controls. Further, a significant association with advanced tumour stage, tumour necrosis, and high Fuhrman grade was observed. The $20 \mathrm{~S}$ proteasome serum levels were independently associated with DSS and increased the predictive accuracy of a standard prognostic model. Finally, this study generates the hypothesis that this marker may be helpful for the identification of patients with metastatic disease, who will most likely benefit from sunitinib treatment.

In our study, circulating $20 \mathrm{~S}$ proteasome levels were elevated in patients with CCRCC. Lavabre-Bertrand et al (2001) were the first that assessed the role of this serum marker in cancer patients. Here, patients with solid tumours, myeloproliferative, and myelodysplastic syndromes had significantly higher levels than healthy donors. In the last years, similar data have been observed in other malignancies, including multiple myeloma (Jakob et al, 2007), breast cancer (Hoffmann et al, 2011), ovarian cancer (Heubner et al, 2011), and malignant melanoma (Stoebner et al, 2005). The source of this elevated $20 \mathrm{~S}$ proteasome levels is unclear. It has been suspected that the proteasome levels originate from both tumour cells and non-malignant cells as a result of an immune reaction. Reinforcing the former concept, the proteasome is overexpressed in RCC and correlates with proliferation (Kanayama et al, 1991). This fact may also explain why $20 \mathrm{~S}$ proteasome serum levels were associated with variables of tumour burden and differentiation, such as tumour size, $M$ stage, and grade. In malignant melanoma, a similar relationship was observed (Stoebner et al, 2005). However, immunohistochemical expression in the tumour did not correlate with circulating proteasome levels in several studies (Heubner et al, 2011; Hoffmann et al, 2011), indicating a role of non-malignant cells and benign diseases. In fact, benign conditions, including vascular, pulmonary, or autoimmune diseases can significantly alter circulating proteasome levels (Egerer et al, 2002; Roth et al, 2005). In our pilot study, we did not investigate the relationship between immunohistochemical staining and circulating proteasome levels; however, this should be done in further studies.

Prediction of CCRCC prognosis and response to targeted therapy is mainly based on clinical and pathological variables. Conventional prognostic factors include performance status, TNM stage, grade, tumour size, and tumour necrosis. As one factor alone is not sufficient to predict prognosis accurately, multiple prognostic factors have been combined into prognostic models and nomograms (Frank et al, 2002; Zisman et al, 2002; Karakiewicz et al, 2007). For example, the SSIGN score combines TNM stage, size, grade, and necrosis for patients with CCRCC. Recent progress in molecular biology has identified novel genetic and protein markers, which may represent additional indicators of patients with biologically aggressive, high-risk RCC (de Martino et al, 2012). The current study identified 20 S proteasome serum levels as such a prognostic biomarker. Several other small studies were thought to identify preoperative prognostic serum markers. Thompson et al (2008) studied preoperative serum levels of B7x in 101 patients with CCRCC. Serum levels were higher in patients with a tumour thrombus, positive lymph nodes, and distant metastases. In a study on 74 patients, preoperative VEGF-A levels were independently associated with poor DSS (Klatte et al, 2007a). $\mathrm{Li}$ et al (2008) showed that higher preoperative carbonic anhydrase-9 levels correlate with diminished recurrence-free survival. However, these markers were not validated externally and predictive accuracies were not assessed.

In this regard, the predictive accuracy of prognostic factors is generally assessed by the concordance index. Prognostic factors may be statistically significant in multivariable analysis, but it is possible that they do not add predictive information and may be therefore less relevant. Previous studies showed a predictive accuracy of the SSIGN score of $82 \%$ and $90 \%$, respectively (Frank et al, 2002; Ficarra et al, 2006), which is in accordance with the current report. Of utmost importance, the predictive accuracy of the SSIGN score increased significantly when 20 S proteasome serum levels were introduced as a variable. If validated by other groups, 20S proteasome serum levels may assist in postoperative risk stratification and may therefore allow risk-adjusted follow-up, or identify patients that should be included in adjuvant clinical trials.

It is similarly crucial to predict response to systemic therapy in patients with metastatic disease; however, this ability is limited by conventional prognostic models. In many patients, sunitinib will represent the first-line therapy. Attempts have been made to identify molecular markers predicting response to sunitinib. Paule et al (2010) evaluated the expression of 16 biomarkers in the primary tumour and identified soluble VEGF isoforms as predictors of response to sunitinib. Our data on 22 patients receiving sunitinib generate the hypothesis that circulating $20 \mathrm{~S}$ proteasome serum levels may be another predictive marker. We analysed pre-operative samples, but not the levels following surgery, and thus, before the start of sunitinib. However, this should be done in a future study testing this hypothesis. The $20 \mathrm{~S}$ proteasome serum levels may be further tested as a marker predicting response to bortezomib, as bortezomib reversibly inhibits the $20 \mathrm{~S}$ proteasome subunit. Of 37 patients with metastatic RCC that were evaluated in a phase II trial, 4 (11\%) achieved a partial response and $11(38 \%)$ stable disease (Kondagunta et al, 2004). This clinical benefit rate may increase with proper patient selection according to the $20 \mathrm{~S}$ proteasome serum levels.

Study limitations have to be acknowledged and addressed. As a single centre pilot study, few patients with limited follow-up were included. Sub-group analysis, for example, from patients receiving sunitinib therapy was limited by sample size. However, specific sub-group analyses were beyond the scope of this study. An external validation on large, prospective, multi-institutional cohorts with long-term follow-up will be necessary before this 
marker can be applied in clinical routine. The hypotheses generated, such as the role as a predictive marker for targeted therapy, deserve further investigation.

In summary, we identified circulating $20 \mathrm{~S}$ proteasome as a novel diagnostic and prognostic serum marker for CCRCC. Its routine use may allow better diagnosis, risk stratification, risk-adjusted follow-up, and identification of patients with a greater likelihood of response to sunitinib therapy. External, prospective validation on large cohorts is warranted.

\section{Conflict of interest}

The authors declare no conflict of interest.

\section{REFERENCES}

Corn PG (2007) Role of the ubiquitin proteasome system in renal cell carcinoma. BMC Biochem 8: S4

de Martino M, Klatte T, Haitel A, Marberger M (2012) Serum cell-free DNA in renal cell carcinoma: a diagnostic and prognostic marker. Cancer 118: $82-90$

Egerer K, Kuckelkorn U, Rudolph PE, Ruckert JC, Dorner T, Burmester GR, Kloetzel PM, Feist E (2002) Circulating proteasomes are markers of cell damage and immunologic activity in autoimmune diseases. $J$ Rheumatol 29: $2045-2052$

Ferlay J, Shin HR, Bray F, Forman D, Mathers C, Parkin DM (2010) Estimates of worldwide burden of cancer in 2008: GLOBOCAN 2008. Int J Cancer 127: 2893-2917

Ficarra V, Martignoni G, Lohse C, Novara G, Pea M, Cavalleri S, Artibani W (2006) External validation of the Mayo Clinic Stage, Size, Grade and Necrosis (SSIGN) score to predict cancer specific survival using a European series of conventional renal cell carcinoma. J Urol 175: 1235 - 1239

Frank I, Blute ML, Cheville JC, Lohse CM, Weaver AL, Zincke H (2002) An outcome prediction model for patients with clear cell renal cell carcinoma treated with radical nephrectomy based on tumor stage, size, grade and necrosis: The SSIGN score. J Urol 168: 2395-2400

Gore ME, Larkin JM (2011) Challenges and opportunities for converting renal cell carcinoma into a chronic disease with targeted therapies. Br J Cancer 104: 399-406

Groll M, Berkers CR, Ploegh HL, Ovaa H (2006) Crystal structure of the boronic acid-based proteasome inhibitor bortezomib in complex with the yeast 20S proteasome. Structure 14: 451-456

Heubner M, Wimberger P, Dahlmann B, Kasimir-Bauer S, Kimmig R, Peters J, Wohlschlaeger J, Sixt SU (2011) The prognostic impact of circulating proteasome concentrations in patients with epithelial ovarian cancer. Gynecol Oncol 120: 233-238

Hoffmann O, Heubner M, Anlasik T, Winterhalter M, Dahlmann B, Kasimir-Bauer S, Kimmig R, Wohlschlaeger J, Sixt SU (2011) Circulating $20 \mathrm{~S}$ proteasome in patients with non-metastasized breast cancer. Anticancer Res 31: 2197-2201

Jakob C, Egerer K, Liebisch P, Turkmen S, Zavrski I, Kuckelkorn U, Heider U, Kaiser M, Fleissner C, Sterz J, Kleeberg L, Feist E, Burmester GR, Kloetzel PM, Sezer O (2007) Circulating proteasome levels are an independent prognostic factor for survival in multiple myeloma. Blood 109: 2100-2105

Kanayama H, Tanaka K, Aki M, Kagawa S, Miyaji H, Satoh M, Okada F, Sato S, Shimbara N, Ichihara A (1991) Changes in expressions of proteasome and ubiquitin genes in human renal cancer cells. Cancer Res 51: $6677-6685$

Karakiewicz PI, Briganti A, Chun FK, Trinh QD, Perrotte P, Ficarra V, Cindolo L, De la Taille A, Tostain J, Mulders PF, Salomon L, Zigeuner R, Prayer-Galetti T, Chautard D, Valeri A, Lechevallier E, Descotes JL, Lang H, Mejean A, Patard JJ (2007) Multi-institutional validation of a new renal cancer-specific survival nomogram. J Clin Oncol 25: $1316-1322$

Klatte T, Bohm M, Nelius T, Filleur S, Reiher F, Allhoff EP (2007a) Evaluation of peri-operative peripheral and renal venous levels of pro- and anti-angiogenic factors and their relevance in patients with renal cell carcinoma. BJU Int 100: 209-214

Klatte T, Pantuck AJ (2008) Molecular biology of renal cortical tumors. Urol Clin North Am 35: $573-580$

Klatte T, Seligson DB, Riggs SB, Leppert JT, Berkman MK, Kleid MD, Yu H, Kabbinavar FF, Pantuck AJ, Belldegrun AS (2007b) Hypoxia-inducible factor 1 alpha in clear cell renal cell carcinoma. Clin Cancer Res 13: $7388-7393$

Kondagunta GV, Drucker B, Schwartz L, Bacik J, Marion S, Russo P, Mazumdar M, Motzer RJ (2004) Phase II trial of bortezomib for patients with advanced renal cell carcinoma. J Clin Oncol 22: $3720-3725$

Lavabre-Bertrand T, Henry L, Carillo S, Guiraud I, Ouali A, Dutaud D, Aubry L, Rossi JF, Bureau JP (2001) Plasma proteasome level is a potential marker in patients with solid tumors and hemopoietic malignancies. Cancer 92: $2493-2500$

Li G, Feng G, Gentil-Perret A, Genin C, Tostain J (2008) Serum carbonic anhydrase 9 level is associated with postoperative recurrence of conventional renal cell cancer. J Urol 180: 510-514

Ljungberg B, Hanbury DC, Kuczyk MA, Merseburger AS, Mulders PF, Patard JJ, Sinescu IC (2007) Renal cell carcinoma guideline. Eur Urol 51: $1502-1510$

Paule B, Bastien L, Deslandes E, Cussenot O, Podgorniak MP, Allory Y, Naimi B, Porcher R, de La Taille A, Menashi S, Calvo F, Mourah S (2010) Soluble isoforms of vascular endothelial growth factor are predictors of response to sunitinib in metastatic renal cell carcinomas. PLoS One 5: e10715

Powles T, Chowdhury S, Jones R, Mantle M, Nathan P, Bex A, Lim L, Hutson T (2011) Sunitinib and other targeted therapies for renal cell carcinoma. Br J Cancer 104: $741-745$

Procopio G, Verzoni E, Bracarda S, Ricci S, Sacco C, Ridolfi L, Porta C, Miceli R, Zilembo N, Bajetta E (2011) Sorafenib with interleukin-2 vs sorafenib alone in metastatic renal cell carcinoma: the ROSORC trial. Br J Cancer 104: $1256-1261$

Roth GA, Moser B, Krenn C, Roth-Walter F, Hetz H, Richter S, Brunner M, Jensen-Jarolim E, Wolner E, Hoetzenecker K, Boltz-Nitulescu G, Ankersmit HJ (2005) Heightened levels of circulating 20S proteasome in critically ill patients. Eur J Clin Invest 35: 399-403

Sobin LH, Gospodarowicz MK, Wittekind C (2009) Urological tumours kidney. In TNM Classification of Malignant Tumours 7th edn, pp 255-257. Wiley-Blackwell: Oxford

Stoebner PE, Lavabre-Bertrand T, Henry L, Guiraud I, Carillo S, Dandurand M, Joujoux JM, Bureau JP, Meunier L (2005) High plasma proteasome levels are detected in patients with metastatic malignant melanoma. Br J Dermatol 152: $948-953$

Szerafin T, Hoetzenecker K, Hacker S, Horvath A, Pollreisz A, Arpad P, Mangold A, Wliszczak T, Dworschak M, Seitelberger R, Wolner E, Ankersmit HJ (2008) Heat shock proteins 27, 60, 70, 90alpha, and 20S proteasome in on-pump versus off-pump coronary artery bypass graft patients. Ann Thorac Surg 85: 80-87

Thompson RH, Zang X, Lohse CM, Leibovich BC, Slovin SF, Reuter VE, Cheville JC, Blute ML, Russo P, Kwon ED, Allison JP (2008) Serumsoluble $\mathrm{B} 7 \mathrm{x}$ is elevated in renal cell carcinoma patients and is associated with advanced stage. Cancer Res 68: 6054-6058

Tisdale MJ (2003) Pathogenesis of cancer cachexia. J Support Oncol 1: $159-168$

Voges D, Zwickl P, Baumeister W (1999) The 26S proteasome: a molecular machine designed for controlled proteolysis. Annu Rev Biochem 68: $1015-1068$

Zisman A, Pantuck AJ, Wieder J, Chao DH, Dorey F, Said JW, DeKernion JB, Figlin RA, Belldegrun AS (2002) Risk group assessment and clinical outcome algorithm to predict the natural history of patients with surgically resected renal cell carcinoma. J Clin Oncol 20: $4559-4566$

This work is published under the standard license to publish agreement. After 12 months the work will become freely available and the license terms will switch to a Creative Commons Attribution-NonCommercial-Share Alike 3.0 Unported License. 